\title{
Tangence
}

\section{La violence de l'imaginaire}

Gramsci et Sorel

\section{Terry Cochran}

Numéro 63, juin 2000

Fictions et politique

URI : https://id.erudit.org/iderudit/008182ar

DOI : https://doi.org/10.7202/008182ar

Aller au sommaire du numéro

Éditeur(s)

Presses de l'Université du Québec

ISSN

0226-9554 (imprimé)

1710-0305 (numérique)

Découvrir la revue

Citer cet article

Cochran, T. (2000). La violence de l'imaginaire : gramsci et Sorel. Tangence, (63), 55-73. https://doi.org/10.7202/008182ar

Ce document est protégé par la loi sur le droit d'auteur. L'utilisation des services d'Érudit (y compris la reproduction) est assujettie à sa politique d'utilisation que vous pouvez consulter en ligne.

https://apropos.erudit.org/fr/usagers/politique-dutilisation/ 


\section{La violence de l'imaginaire. Gramsci et Sorel Terry Cochran, Université de Montréal}

\section{Politique, fiction, image et fondation}

Dans un monde constamment traversé par une pléthore de représentations symboliques qui réveillent et provoquent des désirs, des pensées et des actions aussi réels que virtuels, la tâche visant à démarquer la politique de la fiction est irréalisable. Ainsi les images symboliques ou emblématiques, souvent sous la forme de personnifications, ont littéralement structuré la mentalité collective de la modernité, offrant un moyen idéal de fusionner la conception de la communauté et sa réalisation territoriale, culturelle ou religieuse. Les exemples de cette opération figurative sont nombreux et comprennent la figure du Christ, notamment dans De imitatione Christi de Thomas Kempis dont s'inspire saint Ignace de Loyola en fondant la Société de Jésus; la représentation littéraire de Vasco de Gama dans Les Lusiades de Camões, image jetant les bases de la littérature portugaise et de la conscience étatique; la figure de Jeanne d'Arc devenue l'incarnation du rassembleur par excellence de la France; ainsi que des images ou des notions plus abstraites, comme celle de "progrès", qui sous-tend la plupart des récits historiques occidentaux depuis le $\mathrm{XVIII}^{\mathrm{e}}$. Dans ce sens, les images jouent un rôle fondamental de catalyseur dans la représentation du monde "réel", d'un monde meilleur avec ses rêves de transformation; enfin, elles jouent un rôle dans la compréhension de l'histoire ainsi que dans les projets potentiels visant à métamorphoser le domaine politique.

Les images littéraires — ou, plutôt, textuelles — restent un véhicule essentiel pour la diffusion des figures de la pensée, mais l'économie instaurée par l'écriture prolifère dans d'autres médias et l'écriture elle-même n'est plus liée exclusivement au livre, au papier ou à une matière de reproduction palpable. En outre, les images excèdent très souvent le littéraire, l'écrit, et circulent sous une forme déjà visualisée qui n'exige plus de médiation linguistique. Néanmoins, malgré l'extension monstrueuse des images, inondant le globe selon une immédiateté multimédiatique, l'entrelacement de "fiction" et de "politique" persiste, même si la puissance de ces amalgames éventuels a augmenté d'une manière 
56

exponentielle. C'est dans ce contexte que la question du pouvoir fondateur de la fiction, du littéraire ou, plus précisément, de la représentation sous une forme quelconque, a la plus grande résonance. En fin de compte, la question de la fondation relève de la force de l'image, de l'imaginaire, et de son incarnation représentée. Il en va de même pour la collectivité qui se reconnaît dans une image et trouve son fondement dans une représentation qui la constitue comme protagoniste historique. La réflexion sur cette problématique se révèle extrêmement abstraite et difficile, comme l'indique même le concept de "fondation", qui ressemble davantage à une image qu'à un concept. Pendant toute la modernité, la fiction fondatrice a été tout autant "politique" que l'action dite "exécutive". Au $\mathrm{xx}^{\mathrm{e}}$ siècle, moment historique de grands mouvements de masse, de nombreux penseurs politiques et littéraires ont réfléchi sur la nécessité d'identifier une image rassembleuse susceptible de cristalliser un mouvement politique, et de produire une prise de conscience collective. Dans ce contexte, les formulations d'Antonio Gramsci ont été décisives.

\section{Gramsci et Machiavel}

Les propositions de Gramsci ont profondément marqué la théorie et la pratique sociopolitiques après la Deuxième Guerre Mondiale. Malgré la distance qui sépare ses analyses du monde contemporain, elles demeurent pertinentes quant aux aspects principaux de toute image fondatrice et des présupposés qui l'accompagnent. En fait, Gramsci a spécifiquement théorisé la consolidation du parti politique comme un problème conceptuel. En vue de quoi et dans quelles conditions pourrait-on concevoir un ensemble ou une collectivité politique? Autrement dit, comment penser le moteur du parti politique afin de canaliser ses forces historiques? Ses considérations sur cette question, qui visait le contexte philosophique et politique à l'époque du fascisme, commencent avec une discussion des théories politiques de Machiavel, qui a été le premier à saisir et à articuler les paramètres de l'État moderne ${ }^{1}$.

En discutant les analyses de Machiavel, Gramsci remarque:

1. Antonio Gramsci, "Petites notes sur la politique de Machiavel", Cahier 13, Cabiers de prison, Paris, Gallimard, 1978. J'indiquerai les pages de ce texte entre parenthèses après les citations. 
Entre l'utopie et le traité scolastique, formes sous lesquelles se présentait la science politique jusqu'à Machiavel, celui-ci a donné à sa conception la forme imaginative et artistique, grâce à laquelle l'élément doctrinal et rationnel se trouve personnifié par un condottiere, qui représente de façon plastique et "anthropomorphique" le symbole de la volonté collective. (p. 353)

Traçant la figure du prince comme représentation de l'action collective, Machiavel a introduit une autre façon de raisonner, qui signale une nouvelle économie politique que l'histoire subséquente a nommée la "modernité". Avec pour résultat sans doute un genre de pensée basée sur l'image, sur la mise en image d'une force politique et historique, qui fusionne l'action réelle avec sa représentation idéale. Dans les commentaires de Gramsci, ce "genre" de pensée se différencie à la fois du discours du traité et de la projection utopique. En tant que discours établis qui ont fortement imprégné la tradition moderne, l'utopie et le traité impliquent des théories respectives de l'action et des conditions menant à sa réalisation. À sa façon, l'utopie laïcise le jugement dernier de la divinité chrétienne et projette une image vers l'avenir; ce futur, plus ou moins éloigné, sert de référent idéal et permet la formation d'un groupe ou d'une communauté qui attend l'accomplissement de cette projection. Au fond, l'utopie est une question de foi, même si les membres qui partagent cet idéal s'engagent consciemment à en diminuer l'attente. En revanche, le traité, ancré dans les fictions de la connaissance objective, décrit les conditions, les facteurs et les principes qui influeraient sur une action éventuelle ou qui ont contribué à une conclusion ou à une certaine série d'événements. Il sépare, d'une façon nette, l'objet, l'esprit observateur et l'analyse raisonnée.

Néanmoins, selon les interprétations de Gramsci, la figure du prince ou du condottiere n'est pas irrationnelle; en tant que personnification ou image "anthropomorphique", cette figure appartient au domaine de la fiction, de la représentation idéale. Le prince est simultanément l'expression et la représentation du mouvement collectif que la figure incarne. La personnification et la prosopopée sont des tropes très particuliers dans les études littéraires, justement parce qu'ils fournissent les moyens - sorte de mécanisme rhétorique - de penser ce qui est impensable en soi. Une collectivité quelconque ne peut sans doute même pas devenir le "sujet" d'une phrase sans une opération rhétorique, sans 
l'utilisation d'une figure de la pensée, qui unit dans une seule image des individus disparates qui, au départ, n'agissent jamais dans le même esprit. Dans cette optique, la personnification est l'épine dorsale du langage; c'est pourtant Machiavel qui transpose cette mise en image dans la sphère politique. En se référant au symbole - trope qui traditionnellement décrivait l'intégration inébranlable entre l'expression et l'exprimé —, Gramsci souligne l'enchevêtrement du protagoniste historique et de la pensée qui le conçoit. Cette manière d'aborder des secteurs d'une multitude informe, de la saisir en bloc, articule le processus de figuration politique qui sera le moteur de la modernité.

Dans le discours de Gramsci, ainsi que dans son interprétation de Machiavel, la figuration de la collectivité va toujours de pair avec la fondation politique. En refusant de séparer la théorie de ses conséquences pratiques, c'est-à-dire les interprétations de leurs implications politiques, Gramsci réclame ce processus de mise en image, de figuration du corps politique, pour fonder un mouvement social. Encore une fois, il élabore cette idée à partir de la figure de Machiavel: "Tout au long de son petit livre Machiavel traite de ce que doit être le Prince pour pouvoir conduire un peuple à la fondation d'un nouvel État [...]" (354). De même qu'un État - un stato, mot que Machiavel utilise pour la première fois dans son acception moderne - est une institution fondée, qui comprend une administration et une armée réunies autour d'un "prince", de même le peuple, personnifié par la figure du prince qui ne l'incorpore pas en chair et en os, agit comme un individu seul pour prendre le pouvoir, pour fonder l'État à nouveau, mais sur d'autres bases. La nouveauté du propos machiavélien concerne le superflu de l'individu empirique: il faut imaginer, rendre en image, un collectif qui, en tant qu'image, agit comme une personne singulière. Dans cette perspective collective, la possibilité de fonder, de concevoir la fondation comme un geste qui résulte des actes d'un sujet, exige l'image idéalisée d'une figure immatérielle. Le prince de Machiavel, du moins pour Gramsci, est autant figure que prince, même si l'image est calquée sur le Valentinois (Cesare Borgia).

La conscience dite moderne - politique, historique, subjective, culturelle - repose sur la personnification. Elle fait partie du sédiment de l'esprit et constitue un acquis idéal dans un monde dont la pensée est de moins en moins empirique, de plus en plus virtuelle. Or la personnification, la figuration collective, relève de 
la fiction. Quant à la figure du prince, les analyses de Gramsci sont tout particulièrement révélatrices:

Le procès de formation d'une volonté collective déterminée, pour une fin politique déterminée, se trouve représenté non pas au moyen de pédantes discussions et de pédantes classifications de principes et de critères d'une méthode d'action, mais par les qualités, les traits caractéristiques, les devoirs, les nécessités d'une personne concrète, ce qui permet de mettre en œuvre l'imagination artistique de celui qu'on veut convaincre et ce qui donne une forme plus concrète aux passions politiques. (353)

L'image représente le processus de consolidation d'une volonté collective parce qu'elle crée la possibilité de visualiser un acteur concret, servant en quelque sorte de peau pour une masse amorphe et sans contenant. Cette image est davantage une incarnation idéale qu'une représentation simple: elle projette une forme dans l'esprit à laquelle on peut attribuer une volonté, une conscience, un visage avec des expressions émotives, des bras pour saisir les objets du monde, des jambes pour se déplacer ou pour donner des coups de pied, et, bien sûr, des devoirs historiques. Par ailleurs, l'image ne se réduit pas au plan idéaliste: au lieu de créer les sentiments qu'elle convoque, elle incarne les passions collectives qui existent déjà, mais d'une façon nébuleuse. Ainsi, la personnification, appliquée au domaine politique, se montre extrêmement efficace et surtout très littéraire; ce qui, dans le texte gramscien, est indiqué par l'évocation de ses aspects "artistiques". L'art, l'artifice, la fiction tracent des tableaux qui excèdent le réel en l'inscrivant, qui le produisent en le saisissant. La représentation de la volonté collective prend, en même temps, la forme d'une écriture et d'une lecture; la persona qu'on invente pour sentir et englober les passions politiques s'insère dans une narration qui se déploie historiquement. La fiction qui la véhicule convainc les lecteurs qui s'identifient avec le protagoniste principal. Tout cela au nom de la fondation éventuelle, qui occupe une place privilégiée dans ce paradigme historique.

Mais, comme tous les penseurs engagés dans une praxis un faire contemporain -, Gramsci considère le discours machiavélien pour mieux cerner son présent. Ancré dans sa contemporanéité, il tâche de déchiffrer les modalités de l'action collective et fondatrice au $\mathrm{xx}^{\mathrm{e}}$ siècle. À travers les siècles d'élaboration sociopolitique, le prince - en tant que concept, figure et personnage 
60

politique - subit des transformations radicales. Au contraire de Machiavel, qui avait idéalisé la figure du prince à partir d'un individu historique afin de lui donner un sens collectif, Gramsci amorce ses réflexions à un niveau plus abstrait et aborde directement la question de la figuration:

Le prince moderne, le mythe-prince, ne peut être une personne réelle, un individu concret, il ne peut être qu'un organisme, un élément complexe de société dans lequel a commencé déjà de se concrétiser une volonté collective qui s'est reconnue et affirmée en partie dans l'action. Cet organisme est déjà donné par le développement historique et c'est le parti politique, première cellule dans laquelle se concentrent des germes de volonté collective qui tendent à devenir universels et totaux. (355-356)

Ce prince - moderne comme le $\mathrm{xx}^{\mathrm{e}}$ siècle européen et comme l'idéologie de la modernisation que l'occident exporte - est d'emblée une figure. Pourtant cette figure, ce qu'elle incarne, n'est que partiellement esquissée: le corps collectif jette son ombre, mais a besoin d'un visage. En somme, c'est une institution idéale en voie de se fonder. Mais dès qu'on interroge le processus par lequel se rassemblent des individus nécessairement divergents, on tombe immédiatement dans la tautologie, comme le discours gramscien le démontre. Dans le texte de Gramsci - et cela vaut pour n'importe quel penseur qui vise la fondation politique - , cette multiplicité se manifeste comme un organisme, un être vivant, un corps politique. Dans cette perspective, la lecture du prince machiavélien révèle sa nécessité en jetant les bases encore une fois - d'un raisonnement qui attribue une existence organique à la collectivité.

Néanmoins, ce discours - comme tout discours - est en proie à ses présupposés; pour comprendre l'ampleur de ces idées préconçues, il est indispensable de suivre jusqu'à son terme la logique de cette attribution figurée. En évoquant l'organisme, qui entame une chaîne inévitable de figures, la possibilité de lui imputer une volonté propre est solidement établie. Cette volonté collective - déjà pleinement dans la figuration - commence à se reconnaître, à se saisir comme acteur ou protagoniste. C'est la prise de conscience hégélienne, histoire qui se répète souvent et avec énormément de facilité ; mais, dans ce cas-ci, ce n'est qu'un coin immanent de l'esprit, à savoir un parti politique qui entreprend de refonder, de devenir l'État. Appliquée à un ensemble hétéroclite, la figure de l'organisme nie ses différences constituti- 
ves pour assumer consciemment la volonté qui lui est propre et qui se dévoile dans l'histoire. En tant qu'être vivant, l'organisme grandit dans le temps; le parti politique est sa première manifestation cellulaire (et la notion de cellule reste très présente, même aujourd'hui, dans les descriptions des petits groupes, unités ou cellules révolutionnaires). Ces germes de la volonté, nés au sein de la cellule qu'est le parti, passent à travers une période d'incubation et par des stades embryonnaires de développement, pour arriver à la majorité, à une connaissance de soi. Le parti n'est qu'une partie de l'organisme qui le transcende en devenant enfin adulte; dans le glissement métaphorique de ce passage, l'âge adulte signale le moment où l'organisme devient universel, totalisant ou, effectivement, englobant.

En dernière analyse, je ne veux critiquer ni le discours gramscien, ni ses intentions politiques. Mon insistance sur les propositions de Gramsci, qui formulait un plan d'action sociale en réponse à sa lecture des forces politiques de son époque, porte plutôt sur le paradigme conceptuel qu'il avance et sur la réflexion profonde qui le caractérise. Cette économie politique du discours, qui dépend d'un emmêlement indéfectible entre la figuration, l'élaboration imaginaire et la fondation, jouit d'une hégémonie sans égale dans une modernité dont l'existence exige la constitution de sujets collectifs, de frontières idéales et réelles, de territorialisations massives. Les conséquences de ce "dispositif", en lequel fusionnent des éléments discursifs et politiques, concernent autant la pensée que l'action, autant le plan idéologique que la pratique. D'un côté, la figuration donne forme au sujet collectif et oriente une volonté; de l'autre, le discours où s'insère la figure fournit ses qualités et ses modalités d'action, offrant ainsi un encadrement narratif et historique. Ensemble, ils sous-tendent et expriment les divers déroulements politiques.

Comme le montrent les affirmations gramsciennes qui exemplifient d'une manière très précise les implications de ce dispositif conceptuel, la figuration collective est supposée aboutir à la fondation, à la prise de pouvoir et à l'investissement des structures sociopolitiques, culturelles et discursives. En un mot, l'organisme mûrit: il dépasse ses contraintes spatio-temporelles et devient universel. Dans ce contexte, la question de l'universalisme n'est pas gratuite et se dévoile comme une composante essentielle du paradigme fondateur, qui continue à exercer une force formidable sur l'organisation du savoir. Comme l'attestent les multiples 
62

mouvements contemporains qui visent la fondation mondiale d'un régime commercial, culturel, juridique, politique et militaire, le thème de l'universel réémerge dans un monde en pleine mutation idéologique. Malgré la durée de son hégémonie, il apparaît que cette logique universalisante de la fondation - qui est inévitablement liée à la représentation, à la fiction, à la production figurée - n'a rien d'anachronique. L'universel constitue une figure qui est partout et nulle part, à notre époque dite "planétaire" où un certain discours de la fondation se répand sur le globe entier. Mais quels sont les enjeux de la figure (notamment de l'organisme devenu universel) qui opère la jonction entre la fondation institutionnelle, même "révolutionnaire", et le fondement de la pensée?

\section{Sorel et la violence}

Quel que soit l'isolement de Gramsci, qui consignait ses pensées dans de petits cahiers qui ne seront publiés qu'après sa mort, ces considérations n'ont pas lieu dans un vide et constituent une réflexion sur les problèmes sociohistoriques $\mathrm{du} \mathrm{xx}^{\mathrm{e}}$ siècle. D'ailleurs, sa vision de la figuration et de son efficacité politique s'articule à ce qui est devenu une partie intégrale de notre conception du monde. Dans la perspective actuelle, ce qu'il propose à cet égard semble pertinent; les interrogations spécifiques de sa position ont été entièrement effacées de l'histoire de la pensée. En fait, dans ce cahier sur le prince moderne, à savoir la figure du parti politique, Gramsci évoque à plusieurs reprises les théories de Georges Sorel, auteur de Réflexions sur la violence ${ }^{2}$, un livre qui eut un impact important sur les penseurs politiques avant la deuxième guerre mondiale. L'élaboration du prince moderne, plus qu'une réaction aux théories de Sorel, développe certaines idées du penseur français et polémique contre d'autres qui ne s'inséraient pas du tout dans un cadre fondateur. De toute façon, Sorel — ou plutôt le fantôme de Sorel — était un interlocuteur important en ce qui concerne la renaissance de la figure machiavélienne et pour l'interprétation de la figuration politique en général.

2. Georges Sorel, Réflexions sur la violence, Paris, Librairie des "Pages libres", 1908. J'indiquerai les pages de ce texte entre parenthèses après les citations. J'indiquerai les pages de ce texte entre parenthèses après les citations. Pour une version numérisée de l'édition M. Rivière 1921, voir l'adresse URL: http://gallica.bnf.fr/scripts/ConsultationTout.exe?O=n089698. 
Le texte gramscien ne cache ni sa dette épistémologique à l'égard de Sorel ni son mépris pour certains de ses jugements politiques. La notion de ce que j'appelle ici "la figure" occupe une place privilégiée dans ce livre sur la violence et Gramsci la lie explicitement à sa propre conception du prince: "Le Prince de Machiavel pourrait être étudié comme une illustration historique du "mythe" sorélien, c'est-à-dire d'une idéologie politique [...] conçue comme une création de l'imagination concrète qui travaille sur un peuple dispersé et pulvérisé dans le but d'en susciter et d'en organiser la volonté collective" (353-354). En d'autres termes, le prince est une idée, une personnification qui devient un point de référence idéal, un point de jonction dans une idéologie comprise comme une "configuration d'idées". Ce passage souligne à nouveau le travail de l'imagination, d'une part, et l'aspect rassembleur de la figure, d'autre part. La figure, littéralement, fomente et canalise la volonté collective dans une opération qui franchit le fossé entre l'action historique et la réflexion. C'est l'idée centrale que Gramsci prend de Sorel et qu'il utilise dans sa lecture originale du prince machiavélien. L'imagination, à savoir le pouvoir créateur de la fiction et de ses moyens figurés, rassemble des tendances concrètes et les "focalise", ainsi qu'une loupe prend les rayons du soleil afin d'en concentrer l'énergie sur un point donné.

Pourtant, la conception sorélienne a des corollaires historiques et discursifs dont il faut tenir compte afin d'expliciter le but rhétorique et politique du prince moderne comme production imaginaire. Les divergences entre les présupposés historiques de Gramsci et ceux de Sorel concernent justement les modalités de cet imaginaire ainsi que le rôle qu'il joue en suscitant des effets historiques, des sentiments collectifs. Malgré le fait que les écrits de Sorel visent souvent une intervention historique ponctuelle qu'on qualifierait aujourd'hui d' "engagée", si on peut encore utiliser un terme si démodé —, ses formulations sont très précises, bien que provocantes. Sa notion de l'imaginaire a de fortes implications pratiques:

[L]es hommes qui participent aux grands mouvements sociaux se représentent leur action prochaine sous forme d'images de batailles assurant le triomphe de leur cause. Je proposais de nommer mythes ces constructions dont la connaissance offre tant d'importance pour l'historien: la grève générale des syndicalistes et la révolution catastrophique de Marx sont des mythes. J'ai donné comme exemples remarquables de mythes ceux qui furent construits par le christianisme primitif, par la réforme, 
64

par la révolution, par les mazziniens; je voulais montrer qu'il ne faut pas chercher à analyser de tels systèmes d'images, comme on décompose une chose en ses éléments, qu'il faut les prendre en bloc comme des forces historiques, et qu'il faut surtout se garder de comparer les faits accomplis avec les représentations qui avaient été acceptées avant l'action.(p. 32-33)

Évidemment, la cohérence de ce que Sorel propose comme analyse de la figuration ne réside pas au niveau des mots, des signifiants. Il faut résister à la tentation de reléguer au domaine du mythe les commentaires de cet essai sur la violence sociale et sur sa nécessité. Le texte ne cesse de passer du mythe à l'image, de la représentation à la construction, tout en essayant de décrire un processus de figuration qui marque, rend possible et guide des actions, tout en n'aboutissant peut-être à rien. En dernière analyse, les affirmations de cet essai portent sur les modes de penser qui se manifestent à la fois d'une façon collective et individuelle.

Dans le texte comme dans la vie, Sorel est un avocat de la grève générale des syndicalistes, une prise de position radicale à l'époque; mais il la propose en tant qu'une image de l'esprit, tout comme les autres images qu'il déclare importantes pour la consolidation des mouvements sociaux. Les mouvements mentionnés couvrent diverses sphères sociales, allant de la religion au communisme, de la politique réformatrice à celle qui se dit révolutionnaire. La figure de la catastrophe, de l'apocalypse, traverse plusieurs de ces domaines sociaux et des mouvements essentiellement opposés: il ne s'agit pas d'une identification du groupe par rapport à l'idée, mais plutôt d'une mobilisation à partir des figures ou des images qui ne sont pas jugées sur la base de leurs résultats concrets. La pensée - celle des mouvements de masse en particulier, mais pas exclusivement - a lieu en vertu d'images, dont on ne peut cependant pas se débarrasser en agissant. En fin de compte, les images ou les figures sont inextricablement attachées au faire, à la possibilité de produire des effets qui n'ont pas de lien direct avec la figure sur le plan du contenu et de ce qu'elle représente. C'est à cet égard qu'un écart s'ouvre entre le statut de l'imaginaire dans Réflexions et le modèle de personnification gramscien. Pour Sorel, ces images ne prennent pas la forme d'une personnification d'un groupe; elles sont nécessaires pour la pensée - spécifiquement celle qui mène à l'action - et ne peuvent pas être résumées par une seule figure fondatrice, par un prince moderne qui serait métahistorique et jetterait les bases 
intellectuelles d'une prise de pouvoir à un moment donné dans un futur indéfini. Le mythe sorélien, figure de la pensée non "idéalisée" et discours imaginé qui garde un rapport étroit à l'acte, n'est qu'un moteur de l'action sans en être l'origine. Il n'est pas l'instauration de la volonté à partir de laquelle on juge l'étendue et le caractère des actions historiques. Au contraire, c'est une passoire, et le courant qui y passe ne mène à aucun lieu précis ou prévu. Enfin, cette conception de la pensée et de son efficace historique résiste au cadre de la fiction fondatrice, même lorsqu'elle revendique un lien politique entre l'imaginaire, la fiction et l'action historique.

Cet essai philosophique sur la violence trace les contours d'une théorie générale de la connaissance qui est axée sur la question des figures de la pensée et sur la fiction discursive qui les amalgame:

Quand nous agissons, c'est que nous avons créé un monde tout artificiel, placé en avant du présent, formé de mouvements qui dépendent de nous. Ainsi notre liberté devient parfaitement intelligible. [...] Ces mondes artificiels disparaissent généralement de notre esprit sans laisser de souvenirs; mais quand des masses se passionnent, alors on peut décrire un tableau, qui constitue un mythe social. (43-44)

Calqué sur sa représentation de l'opération de l'esprit individuel, ce processus de figuration - qui accompagne plutôt que produit les effets sociaux, historiques et politiques - s'applique autant aux mouvements collectifs qu'aux individus. Ce passage esquisse une production de l'imaginaire qui est devenue très familière dans la deuxième moitié $\mathrm{du} \mathrm{xx}^{\mathrm{e}}$ siècle. Les images qui circulent selon cette économie inondent l'imaginaire contemporain qui, sous la forme de la publicité et de la rhétorique visuelle en général, projette des mondes et des produits qui sont là — dans un avenir très proche si nous avons l'argent ou le crédit - pour être habités et être possédés. Dans une société mondialisée comme la nôtre, la liberté se définit en grande partie par le pouvoir d'achat et par les images qui pénètrent au fond de nos âmes; les images établissent, de cette façon, la représentation de notre liberté. Bien que Sorel n'ait pas considéré la question dans cette optique, il décrit le processus de figuration qui s'installe d'une façon hégémonique dans la société technologique. Au niveau individuel, dans le passage du texte sorélien, les images disparaissent, cèdent à d'autres; aujourd'hui, les images laissent souvent un résidu matériel — un 
66

achat, par exemple — qui périra à son tour. Par contre, la "mise en image" à l'échelle collective incarne les passions d'une multitude (une notion qui rappelle certains passages des cahiers de Gramsci) et devient représentable, mémorable; cette présence imaginaire fait son entrée dans l'histoire en devenant un tableau social qui exprime "les tendances les plus fortes d'un peuple, d'un parti ou d'une classe" (177). Au contraire des penseurs qui mettent toute la force politique dans un groupe historique, Sorel essaie de rendre compte du processus de la consolidation collective, et ses remarques visent la conjonction de l'imaginaire et de l'histoire audelà de sa manifestation particulière.

C'est dans ce sens que l'essai élabore une argumentation qui décrit aussi bien la formation et la cohésion momentanées des multitudes que l'établissement d'un mouvement politique qui revendique des pouvoirs fondateurs. La logique de groupe que cette exposition illustre s'applique également à un public quelconque, à un groupe mobile qui se constitue aussi bien autour de certaines questions sociales qu'autour de son identification à certaines marques de voitures ou à des revues électroniques. La réticence de Sorel vis-à-vis de la représentation historique émane de cette même logique, bien qu'il n'en discute pas explicitement: la représentation de l'histoire, en tant que tableau qui concrétise le monde, les passions et les acteurs sociaux, tombe déjà dans l'imaginaire et ne peut pas en sortir. Il faut juger cette représentation selon ce qu'elle produit en tant que tableau, en tant qu'image: "Il faut juger les mythes comme des moyens d'agir sur le présent; toute discussion sur la manière de les appliquer matériellement sur le cours de l'histoire est dépourvue de sens" (180). La conception du mythe, de la figure, de l'imaginaire qu'on trouve articulée dans l'essai de Sorel appartient à la catégorie des "manières de penser" (180). Comme les images, ces "manières" restent immanentes; elles ne peuvent pas transcender l'histoire, pas plus que l'histoire elle-même ne peut dépasser ses représentations.

Pour agir, il faut avoir des images dans l'esprit; mais une série d'images ou de "figures" n'équivaut pas à une autre. Certaines projections, qui ressemblent à des lectures du "réel ", du politique, y compris des acteurs et de leurs rôles potentiels, ouvrent plus ou moins de possibilités pour produire des effets et réaliser "quelque chose", même si l'on ne le sait pas à l'avance. Cette propension à l'action dont les fins sont imprévisibles indique clairement que les images nécessaires au déroulement de la conscience historique 
ne participent pas à l'économie de la maîtrise, c'est-à-dire de la fiction de la subjectivité, déterminante dans notre modernité. Au contraire, "l'expérience nous prouve que des constructions d'un avenir indéterminé dans les temps peuvent posséder une grande efficacité [...]" (177). De même que pour certains récits, certaines narrations historiques empêchent le développement d'une pensée transformatrice et sa mise en ouvre; de même certaines constructions idéales spécifiques créent des mutations tant sur le plan de la pensée qu'au niveau de l'acte. En d'autres termes, la mise en image selon Sorel n'aboutit pas à une prise de pouvoir, comme l'exige le trajet idéal d'un mouvement qui cherche à se fonder et à s'universaliser.

L'abstraction de l'argument sorélien, ce que l'auteur désigne comme son penchant "métaphysique", a tout de même des éléments pratiques et des répercussions concrètes. Le texte de Sorel évoque de nombreux exemples pour illustrer son raisonnement ainsi qu'on peut le lire dans ce bref commentaire historique:

Les premiers chrétiens attendaient le retour du Christ et la ruine totale du monde païen, avec l'instauration du royaume des saints, pour la fin de la première génération. La catastrophe ne se produisit pas, mais la pensée chrétienne tira un tel parti du mythe apocalyptique que certains savants contemporains voudraient que toute la prédication de Jésus eût porté sur ce sujet unique. (177-178)

La puissance de l'image de la fin du monde était telle que, à l'époque, les récits apocalyptiques sont devenus un genre littéraire. Mais cette projection dans l'avenir — qui provoquait de la peur et de l'espoir en même temps - n'avait rien d'utopique. En tant que figure du futur, l'apocalypse ne dépendait pas de la volonté des croyants mais de l'intervention divine. Et même si ce moment n'est pas arrivé, n'a pas eu lieu, son image guidait l'action en mobilisant et canalisant la passion des masses, ainsi que leur foi. La référence aux savants n'est pas gratuite (et l'hostilité générale de Sorel à l'égard des savants et du savoir institutionnalisé est bien connue et très présente dans le livre): ce sont eux qui voulaient rétrospectivement supprimer les autres aspects de l'enseignement du Messie, afin de donner un visage unidimensionnel à la communauté chrétienne.

En ce qui concerne la question de la fiction, du rapport entre la fiction et la fondation, ce jeu entre le passé et le présent est tout particulièrement mis en relief. Dès que la figure de la pensée 
68

se détache du moment historique, est dépassée, la logique de l'action soulignée par Sorel n'est plus opérationnelle. Cela explique pourquoi le modèle de l'esprit proposé par ces réflexions sur la violence demeure à l'abri ou à l'écart des fondations institutionnelles :

Il n'y a point d'épopée nationale de choses que le peuple ne peut se représenter comme reproduisibles dans un avenir prochain; la poésie populaire implique bien plutôt du futur que du passé; c'est pour cette raison que les aventures des Gaulois, de Charlemagne, des croisés, de Jeanne d'Arc ne peuvent faire l'objet d'aucun récit capable de séduire d'autres personnes que des lettrés. (139)

Orientée exclusivement vers le futur, la conscience populaire et il faut encore insister sur le fait qu'il ne s'agit pas d'un groupe particulier qui monopolise le mouvement historique - ne cherche pas des racines idéales dans le passé. Le passé en tant que passé, en tant qu'inscription du passé enfermée dans un coffrefort et surveillée par les gardiens du savoir, ne séduit pas en soi. Pour les couches populaires - c'est-à-dire celles qui, dans le vocabulaire sorélien, ne sont pas bourgeoises, ne sont pas au pouvoir - , le patrimoine littéraire occupe un grand jardin dans le cimetière de la culture. Selon la conception de Sorel, le passé tout court ne relève que de l'institution; pour imaginer le passé, il faut que l'image s'insère dans une projection vers l'avenir. Ce passage renvoie aux narrations "classiques" en vertu du rôle important, voire fondateur, de la fiction - de la "littérature" - dans l'organisation du savoir et dans la formation de l'esprit national. Le travail fondateur de l'institution a toujours lieu après coup. Par exemple, si la poésie populaire devenait "étudiable", à savoir sanctionnée comme objet d'étude, elle ne serait plus dans la logique de l'action que décrit Sorel. Elle serait simplement une autre brique de la fondation.

\section{Gramsci et Sorel}

En faisant le tour de l'image sorélienne, de la figure de la pensée qui se conjugue avec l'acte historique, on revient à notre point de départ, mieux placés cependant pour interroger le rapport entre la fiction et la fondation. Dans son essai, Sorel présente un dispositif de l'image où l'on voit comment les représentations mentales projettent des fictions qui ouvrent la voie pour l'action. 
Mais ce n'est pas une fondation au sens traditionnel du terme; cet imaginaire ouvert n'arrive jamais à se traduire dans une mise en place fondatrice, dans une prise de pouvoir sociale. Le titre du livre, Réflexions sur la violence, suggère d'emblée les tendances générales du projet sorélien qui s'opposent à l'ordre établi, à la stabilité de l'État fondé et fondateur. En revendiquant la violence comme le noyau où se lient l'action et ses représentations sociopsychiques, la philosophie révolutionnaire de Sorel excède la simple provocation. Bien que le texte - rempli de sarcasmes, d'insultes et d'ironie - ait sa propre violence, sa plus grande violence se produit dans sa vision du monde, celle où le dispositif de l'imaginaire confronte directement l'hégémonie des institutions de l'État aussi bien que de la pensée. Effectivement, Sorel s'attaque à ce qu'il appelle la "philosophie bourgeoise" - qui a fini par dominer l'esprit moderne — , selon laquelle "la violence serait un reste de la barbarie et [...] serait appelée à disparaître sous l'influence du progrès des lumières" (99). En d'autres mots, cette idée historique, basée sur le présupposé du progrès et du développement humain, prévoit la disparition graduelle de la violence à mesure que la raison devient de plus en plus universelle.

Même si, comme Sorel le remarque (cf. p. 280), le taux de violence ne cesse d'augmenter (et la violence actuelle serait pour lui inimaginable), le problème profond de la philosophie des lumières concerne l'institutionnalisation de la force qui se cache derrière la douceur des grands principes. Et ce sont les institutions qui, dans le socialisme sorélien, constituent la vraie matière de la critique: "le socialisme est une philosophie de l'histoire des institutions contemporaines" (61). Dans ce contexte de l'institution, de la fondation, cette philosophie de l'intervention historique conçoit alors la violence selon une distinction fort importante:

Tantôt on emploie les termes force et violence en parlant des actes de l'autorité, tantôt en parlant des actes de révolte. Il est clair que les deux cas donnent lieu à des conséquences fort différentes. Je suis d'avis qu'il y aurait grand avantage à adopter une terminologie qui ne donnerait lieu à aucune ambiguité et qu'il faudrait réserver le terme violence pour la deuxième acception; nous dirions donc que la force a pour objet d'imposer l'organisation d'un certain ordre social dans lequel une minorité gouverne, tandis que la violence tend à la destruction de cet ordre. (256-257)

Ce face-à-face entre deux conceptions porte explicitement sur la question de la fondation, de l'ordre social, de l'État et de la force 
qui est simultanément réelle et symbolique, c'est-à-dire physique et idéologique. Dans le cadre sorélien, la violence sociale et figurée donne assaut à cet ordre, vise à le détruire, à le mettre en miettes. Mais cette violence n'est pas exclusivement liée aux actes exécutifs, car les actes émanent aussi de l'ordre de l'imaginaire. Par une attaque frontale qui espère l'emporter en remplaçant une structure de pouvoir par une autre, cette violence ne dépasse pas la phase destructrice. En définitive, il s'agit d'une violence contre la fondation, utilisant tous les moyens disponibles, y compris ceux de la figure, de l'interprétation historique et de la fiction.

Ce dernier aspect de la thèse sorélienne - la violence déchaînée contre le fondement institutionnel et la fondation sociale - a inévitablement créé des controverses parmi les lecteurs de ce texte. Appréciée largement pour sa conception du "mythe" collectif et de l'action sociopolitique, la théorie de Sorel est souvent critiquée pour son anarchisme, et l'admiration pour sa philosophie de la violence est relativement mitigée. D'un côté, par exemple, Walter Benjamin - entre autres - a été visiblement marqué par la conception historique sorélienne et a intégré dans sa philosophie les conséquences épistémologiques de la violence ; de l'autre, Gramsci, qui a pris et réélaboré la figuration collective telle qu'elle est articulée dans le texte de Sorel, était méthodologiquement agacé par le fait que la violence proposée par Sorel ne menait à rien, n'avait pas de but fondateur. Les cahiers de Gramsci, dont les formulations sur la personnification étaient mon point de départ, rendent explicite la problématique, de plus en plus évidente quant à ses implications.

Comme d'habitude dans les cahiers, l'approche est épistémologique et, comme nous l'avons vu, le problème tourne autour des questions de l'imaginaire, de la représentation historique et de la fondation collective. La critique de base que Gramsci avance est simple et facilement articulée: Sorel, ou plutôt la philosophie politique qu'il élabore, n'arrive pas à manifester une "compréhension du parti politique" (354). Dans le contexte des liens entre le mot, la figure et le mouvement, cette phrase a une résonance qui excède le plan de la description. Elle constate que le mythe sorélien, fiction fournissant la représentation de l'action politique collective, ne permet pas la personnification du parti, ne devient pas une figure transhistorique qui pourrait s'imposer sous une forme institutionnelle et même étatique. De surcroît, Gramsci voit les propos de Sorel comme "une activité [...] de caractère né- 
gatif et préliminaire (le caractère positif ne résidant que dans l'accord réalisé dans les volontés associées), une activité qui ne prévoit pas d'avoir une phase "active et constructive" "(354-55). C'est la négation pure dont rien ne renaît. Cette destruction ne présente pas de moyens pour passer au stade de l'accord des volontés, ce qui est la condition exigée pour la construction "positive". Selon Gramsci, qui pariait toute sa philosophie sur le jeu de la fondation, le positif n'existe pas autrement, c'est-à-dire sans une transition de la destruction à la construction. Ce raisonnement conduit à une incohérence étrange au niveau du discours: Sorel décrit une activité qui n'exclut pas de moment actif de construction. Enfin, le concept même d'action est inextricablement lié à la fondation éventuelle. Sans cet élément, l'activité est vide et sans issue et ne mérite même pas d'être le concept que le mot revendique.

De toute façon, si l'on raisonne à partir de la terre ferme, le problème - l'inaction révolutionnaire de Sorel - ne réussit pas à outrepasser les sables mouvants de la figuration. Sorel ne "comprend" pas le parti politique parce que sa manière d'aborder la figuration, ainsi que les mythes ou les fictions qu'il soulève comme possibles, n'encadre pas un accord collectif des volontés, ne provoque pas une prise de connaissance. Au lieu de devenir une force historique, l'acteur sociopolitique détruit, devient violent, reste un agent provocateur et rien de plus. En somme, le problème de Sorel est de l'ordre de la figure; s'il n'est pas un malade imaginaire, il a une maladie de l'imaginaire. Ainsi, Gramsci s'interroge :

Mais un mythe peut-il être "non constructif", peut-on imaginer, dans l'ordre des intuitions de Sorel, que puisse être producteur d'efficace un instrument qui laisse la volonté collective dans sa phase primitive et élémentaire, celle de sa simple formation, par distinction (par "scission") même violente, c'est-à-dire par la destruction des rapports moraux et juridiques existants? (355)

Question rhétorique qui donne la réponse en posant la question: non. De même que Gramsci nie l'activité de la destruction sans phase positive, de même il conteste la possibilité d'un mythe d'une figure sociopolitique - qui ne soit déjà en passe de se fonder. Il ne peut imaginer un producteur d' "efficace" qui demeure en deçà de la construction, c'est-à-dire en plein milieu de la violence perpétuelle et de la lutte contre la stabilité, l'apaisement idéologique et la force établie. 
Ce conflit insondable entre deux conceptions du monde place le politique et la fiction, l'action historique et sa figuration, dans des constellations différentes. Pour Gramsci, le politique est fondateur par définition; les projections discursives, les mondes imaginaires peuplés avec des acteurs, mobilisent et conduisent la pensée collective. Par contre, la philosophie sorélienne décrit la cristallisation immédiate du collectif, les tableaux imaginaires qui se concrétisent en se réalisant et les volontés qui tendent à la destruction comme fin et non pas comme moyen. Ces deux visions sociopolitiques imputent à l'imaginaire - à la fiction, en l'occurrence - un pouvoir important. Néanmoins, malgré toutes les divergences, les deux constellations conceptuelles ont un élément commun : la figuration dépend de la volonté des êtres humains et de leurs rassemblements, quelle qu'en soit la durée ou la conséquence.

C'est justement dans cette conjoncture qu'un autre lecteur des Réflexions sur la violence déplace le problème sur un autre terrain. Écrits dans la période qui sépare Gramsci et Sorel, les commentaires de Benjamin ouvrent un gouffre épistémologique qui vide la figure de son volontarisme humain. Benjamin radicalise le néant qui menace le projet sorélien; soit parce qu'il voit les tendances fondatrices de toute théorie qui privilégie la volonté, soit parce qu'il veut dégager le problème de son ancrage dans l'histoire empirique. Son argument, qui porte sur l'institution étatique et sa signification pour la philosophie du droit, se termine par cette observation:

Mais il faut rejeter toute violence mythique, la violence fondatrice du droit, qu'on peut appeler violence gouvernante. Il faut rejeter aussi la violence conservatrice du droit, la violence gouvernée qui est à son service. La violence divine, qui est insigne et sceau, non point jamais moyen d'exécution sacrée, peut être appelée souveraine ${ }^{3}$.

La violence divine n'a pas de but, n'est pas un moyen de réaliser une action au nom d'une fin, d'un concept, d'un ailleurs utopique. C'est l'irruption de l'autre absolu, n'ayant rien à voir avec la

3. "Pour une critique de la violence", traduction par Maurice de Gandillac, L'homme, le langage et la culture, Paris, Denoël Gonthier, 1974, cité par Jacques Derrida, dans Force de loi, Paris, Galilée, 1994, p. 133-135. Le livre de Derrida discute plusieurs aspects importants de l'essai de Benjamin et ce, dans un cadre heideggerien. 
volonté des humains qui sont incapables de le connaître en tant que tel. Souveraineté méconnaissable, tout ce qui reste à faire est de l'inscrire, insigne et sceau, dans une figure. La figure de dieu, le prince benjaminien qui revient perpétuellement, ne réussit jamais à coïncider avec la rupture provoquant sa figuration. Comme les images constituant sa constellation, la fiction ne peut pas se débarrasser de l'apocalypse qu'elle incorpore, toujours juste au-delà ou en deçà de la fondation dont on voudrait qu'elle soit le support. Cet imaginaire, trop humain, lutte inévitablement contre son propre anéantissement en véhiculant sans fin la violence sans fond et sans raison. 